\title{
Three-dimensional imaging of random radiation sources
}

\author{
Joseph Rosen and Amnon Yariv \\ California Institute of Technology, M/S 128-95, Pasadena, California 91125
}

Received February 6, 1996

\begin{abstract}
A method to image random three-dimensional source distributions is proposed. We show that, by using a Michelson stellar interferometer in a prescribed fashion, one is able to measure a special form of a threedimensional degree of coherence. The inverse Fourier transform of this coherence function yields the threedimensional intensity distribution of the source as seen from the paraxial far zone. (c) 1996 Optical Society of America
\end{abstract}

The Van Cittert-Zernike theorem ${ }^{1,2}$ establishes that the two-point coherence function in the far field of a quasi-monochromatic spatially incoherent light source is proportional to the Fourier transform of the source's planar intensity distribution. This is the basis of, among others, the long-baseline interferometry in astronomy, in which one uses a measurement of the coherence function to obtain the source intensity distribution. In this Letter the relationship between a three-dimensional (3-D) source and the coherence data obtained in the far paraxial zone is considered. Carter and Wolf ${ }^{3}$ generalized the Van Cittert-Zernike theorem for 3-D sources. According to them, in the case of
$A_{m}(t)$, located a distance $R_{1, m}$ from one point and $R_{2, m}$ from the other, illuminates $P_{1}$ and $P_{2}$ with spherical waves with a complex amplitude proportional to ${ }^{1}$ $A_{m}\left(t-R_{i, m} / c\right) \exp \left[-j k\left(c t-R_{i, m}\right)\right] / R_{i, m}$, where $i=$ 1,$2 ; c$ is the velocity of light; $k=2 \pi / \lambda$; and $\lambda$ is the average wavelength. We also assume that the radiation from each element source is not blocked by other elements. Of course, a source's points whose radiation does not reach the observation points cannot be reconstructed.

The complex degree of coherence, defined by the time average of the normalized product of the fields $E_{1}, E_{2}{ }^{*}$ at points $\mathrm{P}_{1}$ and $\mathrm{P}_{2}$, sampled at the same time, is

$$
\begin{aligned}
\mu\left(P_{1}, P_{2}\right) & =\frac{\left\langle E_{1}(t) E_{2}{ }^{*}(t)\right\rangle}{\left[\left\langle\left|E_{1}(t)\right|^{2}\right\rangle\left\langle\left|E_{2}(t)\right|^{2}\right\rangle\right]^{1 / 2}}=\frac{\sum_{m} \sum_{n}\left\langle A_{m}(t) A_{n}{ }^{*}(t)\right\rangle \exp \left[j k\left(R_{1, m}-R_{2, n}\right)\right]}{\left[\sum_{m}\left\langle A_{m}(t) A_{m}{ }^{*}(t)\right\rangle \sum_{n}\left\langle A_{n}(t) A_{n}{ }^{*}(t)\right\rangle\right]} \\
\cong & \frac{\sum_{n}\left\langle A_{m}(t) A_{m}{ }^{*}(t)\right\rangle \exp \left[j k\left(R_{1, m}-R_{2, m}\right)\right]}{\sum_{m}\left\langle A_{m}(t) A_{m}{ }^{*}(t)\right\rangle}=I_{0}{ }^{-1} \int I_{s}\left(\bar{r}_{s}\right) \exp \left[j k\left(R_{1}-R_{2}\right)\right] \mathrm{d}^{3} r_{s},
\end{aligned}
$$

a quasi-monochromatic spatially incoherent source the complex degree of coherence is proportional to the 3-D Fourier transform of the source's (volume) intensity distribution. Devaney ${ }^{4}$ and LaHaie $^{5}$ considered the inverse problem of 3-D random sources, and Friberg ${ }^{6}$ calculated the radiation efficiency of $3-\mathrm{D}$ partially coherent primary sources. All these authors assumed that the coherence function is available on an envelope that encloses the source from all 3-D directions. In some cases-for example, in stellar observationsthe coherence can be measured only in the paraxial far zone in one essential direction. In what follows we propose a new method to deduce a 3-D source distribution from coherence data obtained in the far paraxial zone in a single plane.

Recently we derived a relationship between the complex degree of coherence in the far paraxial zone and the intensity distribution of a quasi-monochromatic spatially incoherent 3-D source. The main results are summarized briefly as follows: A 3-D light source, shown in Fig. 1 with a coordinate system $\left(x_{s}, y_{s}, z_{s}\right)$, illuminates from the far field two points located in the coordinates system $(x, y, z)$. Without loss of generality, we assume that $z$ and $z_{s}$ are on the same line. Each volume element source $d V_{m}$, with an amplitude where $I_{0}=\int I_{s}\left(\bar{r}_{s}\right) \mathrm{d}^{3} r_{s}, \bar{r}_{s}=\left(x_{s}, y_{s}, z_{s}\right)$, and $I_{s}\left(\bar{r}_{s}\right)$ is the intensity per unit volume of the source, i.e., $I_{s}\left(\bar{r}_{m}\right) d V_{m}=\left\langle A_{m}(t) A_{m}^{*}(t)\right\rangle$. For each spherical wave we approximate the distance $R_{i}$ in the denominator by the average distance $R$. In this derivation we use the quasi-monochromatic assumption ${ }^{2}$ [i.e., $\left.\left\langle A_{m}\left(t-R_{1, m} / c\right) A_{m}^{*}\left(t-R_{2, m} / c\right)\right\rangle=\left|A_{m}\right|^{2}\right]$ and the complete incoherence of the source ${ }^{1}$ [i.e., $\left\langle A_{m}(t) A_{n}^{*}(t)\right\rangle=$ $\delta_{m n}\left|A_{m}\right|^{2}$.

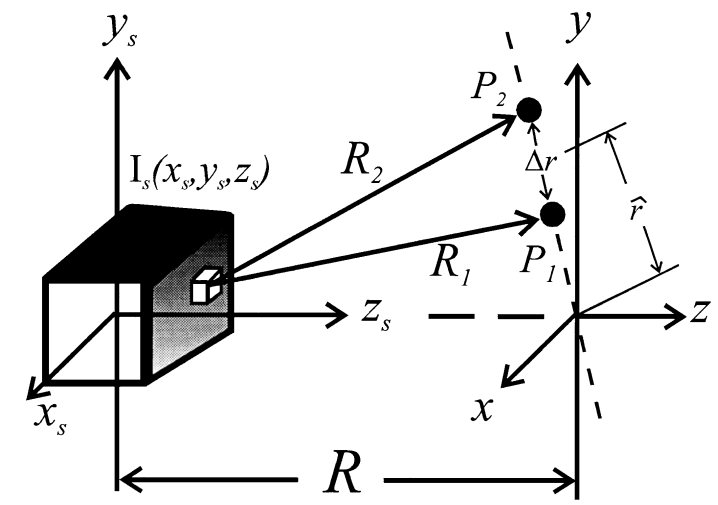

Fig. 1. Schematic showing the calculation of the degree of coherence between $\mathrm{P}_{1}$ and $\mathrm{P}_{2}$.

(C) 1996 Optical Society of America 
The quantity $\left(R_{1}-R_{2}\right)$ in the exponent of relation (1) is approximated under the far-field assumption [i.e., $\left.R \gg \max \left(\left|\bar{r}_{s}\right|\right)\right]$ and under the paraxial-zone assumption [i.e., $R \gg \max (|\bar{r}|)$, where $\bar{r}=(x, y, z)]$. With these approximations the complex degree of coherence between point $\mathrm{P}_{1}$ at $\left(x_{1}, y_{1}, z_{1}\right)$ and point $\mathrm{P}_{2}$ at $\left(x_{2}, y_{2}, z_{2}\right)$ is

$$
\begin{aligned}
\mu\left(P_{1}, P_{2}\right) & \cong C_{0} \int I_{s}\left(\bar{r}_{s}\right) \exp \left\{\frac { - j 2 \pi } { \lambda } \left[\frac{x_{s} \Delta x+y_{s} \Delta y}{R}\right.\right. \\
- & \left.\left.\frac{\Delta z\left(x_{s}{ }^{2}+y_{s}{ }^{2}\right)}{2 R^{2}}+\frac{z_{s}(\hat{x} \Delta x+\hat{y} \Delta y)}{R^{2}}\right]\right\} \mathrm{d}^{3} r_{s},
\end{aligned}
$$

where $C_{0}=I_{0}{ }^{-1} \exp \{j k[\Delta z+(\hat{x} \Delta x+\hat{y} \Delta y) / R]\}, \hat{a}=$ $\left(a_{1}+a_{2}\right) / 2$, and $\Delta a=a_{1}-a_{2}$, with $a$ representing $x, y, z$.

A special case of relation (2) is obtained if both points are in the transverse plane $z=0$ and one of them is fixed at the origin. In this case $\mu(x, y)$ becomes a twodimensional function given by

$$
\begin{aligned}
\mu(x, y)= & C_{0} \int I_{s}\left(\bar{r}_{s}\right) \\
& \times \exp \left\{-\frac{j 2 \pi}{\lambda}\left[\frac{\left(x^{2}+y^{2}\right) z_{s}}{2 R^{2}}+\frac{x x_{s}+y y_{s}}{R}\right]\right\} \mathrm{d}^{3} r_{s} .
\end{aligned}
$$

We showed that $\mu(x, y)$ is formally analogous to the field resulting from coherent propagation through a spherical lens with a focal length $R$ to the plane $(x, y)$ from a 3 -D coherent source $I_{s}\left(\bar{r}_{s}\right)$ located near the front focal plane. Based on this holographic analogy we can reconstruct the 3-D source by first measuring the complex $\mu(x, y)$ [with a Michelson stellar interferometer ${ }^{1,2}$ (MSI), for instance] and then illuminating a transparency $\mu(x, y)$ located at the front focal plane of a spherical lens by a plane wave incident along $z$. The reconstructed 3-D object is obtained around the rear focal point with the appropriate magnification. Such reconstruction, however, does not yield the original source distribution $I_{s}\left(\bar{r}_{s}\right)$ since, based on the holographic imaging analogy, each transverse plane $z_{s}$ contains, in addition to the reconstructed (thus focused) radiation of that plane, unfocused radiation from all the other reconstructed source points.

We now demonstrate a method of overcoming the problem of the unfocused background. A special 3-D degree of coherence yields, by a Fourier transformation, the original 3-D object. The degree of coherence $\mu\left(P_{1}, P_{2}\right)$ is measured with the two points $\mathrm{P}_{1}, \mathrm{P}_{2}$ on the $x y$ plane and on a line passing through the origin (i.e., $\left.x_{1} y_{2}=x_{2} y_{1}\right)$. Under these conditions we use the relation $\hat{r} \Delta r=\hat{x} \Delta x+\hat{y} \Delta y$, where $\hat{r}=\left[\left(x_{1}+x_{2}\right)^{2}+\left(y_{1}+\right.\right.$ $\left.\left.y_{2}\right)^{2}\right]^{1 / 2} / 2$, and $\Delta r=\left[\left(x_{1}-x_{2}\right)^{2}+\left(y_{1}-y_{2}\right)^{2}\right]^{1 / 2}$. If we further change the variable $\hat{r}$ to $q=\hat{r} \Delta r / \Delta r_{\min }$, relation (2) becomes

$$
\begin{aligned}
& \mu(\Delta x, \Delta y, q)=C_{0} \int I_{s}\left(\bar{r}_{s}\right) \\
& \times \exp \left[\frac{-j 2 \pi}{\lambda}\left(\frac{x_{s} \Delta x+y_{s} \Delta y}{R}+\frac{z_{s} q \Delta r_{\min }}{R^{2}}\right)\right] \mathrm{d}^{3} r_{s} .
\end{aligned}
$$

Equation (4) is the fundamental result here, indicating that the degree of coherence along the coordinates $(\Delta x, \Delta y, q)$ (although it is on one transverse plane) is a 3-D Fourier transform of the random source's 3-D intensity distribution as seen from the far paraxial zone. $\mu(\Delta x, \Delta y, q)$ is a degree of coherence obtained from all the possible values of the two-point separation $(\Delta x, \Delta y)$ and the points' center of gravity $\hat{r}$ in which the line connecting the points passes through the origin. The practical meaning of the variable change from $\hat{r}$ to $q$ is that, if for minimum separation $\Delta r_{\text {min }}$ the center of gravity changes in steps of $d_{q}$ until reaching the maximum value of $\hat{r}_{\max }$, then for any other value of $\Delta r$ the center of gravity changes in steps of $d_{q} \Delta r_{\min } / \Delta r$ until reaching the maximum value of $\hat{r}_{\max } \Delta r_{\min } / \Delta r$.

$\mu(\Delta x, \Delta y, q)$ is a function of three orthogonal coordinates and a 3-D Fourier transform (with scaling factors) of the source intensity distribution, now given by

$$
I_{s}\left(\bar{r}_{s}\right) \propto \mathrm{FT}_{3-\mathrm{D}}{ }^{-1}\left\{\mu\left(\frac{\Delta x}{\lambda R}, \frac{\Delta y}{\lambda R}, \frac{q \Delta r_{\min }}{\lambda R^{2}}\right)\right\},
$$

where $\mathrm{FT}_{3-\mathrm{D}}{ }^{-1}$ indicates a $3-\mathrm{D}$ inverse Fourier transform. Relation (5) describes the reconstruction procedure of the source from the measured degree of coherence.

When a degree of coherence is measured by a MSI the actual measured function is a truncated sampled version of $\mu(\Delta x, \Delta y, q)$ and can be expressed as

$$
\begin{aligned}
& \mu^{\prime}\left(\frac{\Delta x}{\lambda R}, \frac{\Delta y}{\lambda R}, \frac{q \Delta r_{\min }}{\lambda R^{2}}\right)=\mu\left(\frac{\Delta x}{\lambda R}, \frac{\Delta y}{\lambda R}, \frac{q \Delta r_{\min }}{\lambda R^{2}}\right) \\
& \times \sum_{k=-K}^{K} \sum_{m=-M}^{M} \sum_{n=-N}^{N} \delta\left(\Delta x-n d_{x}, \Delta y-m d_{y}, q-k d_{q}\right),
\end{aligned}
$$

where $\left(d_{x}, d_{y}, d_{q}\right)$ are the intervals between the samples in the $(\Delta x, \Delta y, q)$ space and $2\left(N d_{x}, M d_{y}, K d_{q}\right)$ is the volume of the measured coherence function. As a result of the sampling, the reconstructed image becomes

$$
\begin{aligned}
I_{s}{ }^{\prime}\left(\bar{r}_{s}\right) \propto \mathrm{FT}_{3-D}{ }^{-1}\left[\mu^{\prime}\left(\frac{\Delta x}{\lambda R}, \frac{\Delta y}{\lambda R}, \frac{q \Delta r_{\min }}{\lambda R^{2}}\right)\right] \\
=\mathrm{FT}_{3-\mathrm{D}}{ }^{-1}\left[\mu\left(\frac{\Delta x}{\lambda R}, \frac{\Delta y}{\lambda R}, \frac{q \Delta r_{\min }}{\lambda R^{2}}\right)\right] \\
*\left[\operatorname{sinc}\left(\frac{2 N d_{x} x_{s}}{\lambda R}\right) \operatorname{sinc}\left(\frac{2 M d_{y} y_{s}}{\lambda R}\right) \operatorname{sinc}\left(\frac{2 K \Delta r_{\min } d_{q} z_{s}}{\lambda R^{2}}\right)\right] \\
\quad * \sum_{k=-\infty}^{\infty} \sum_{m=-\infty}^{\infty} \sum_{n=-\infty}^{\infty} \\
\times \delta\left(x_{s}-\frac{n \lambda R}{d_{x}}, y_{s}-\frac{m \lambda R}{d_{y}}, z_{s}-\frac{k \lambda R^{2}}{\Delta r_{\min } d_{q}}\right),
\end{aligned}
$$

where the asterisk denotes convolution. From relation (7) we see that the width of the sinc functions, which is due to the sampling truncation, determines the resolution limits of the imaging. Therefore the minimum planar distance that can be resolved in the target is $\left(\Delta x_{s, \min }, \Delta y_{s, \min }\right) \approx \lambda R\left(1 / N d_{x}, 1 / M d_{y}\right)$, and the minimum depth interval that can be resolved is $\Delta z_{s, \min } \approx \lambda R^{2} / K \Delta r_{\min } d_{q}=\lambda R^{2} / \hat{r}_{\max } \Delta r_{\min }$. The field of view is determined by the sampling rate, and, for reconstruction of the full object, its size should be less than the distance between two $\delta$ functions. Therefore the object's maximum dimensions that can be reconstructed are $\left(X_{s}, Y_{s}, Z_{s}\right) \approx \lambda R\left(1 / d_{x}, 1 / d_{y}, R / d_{q} \Delta r_{\text {min }}\right)$. 

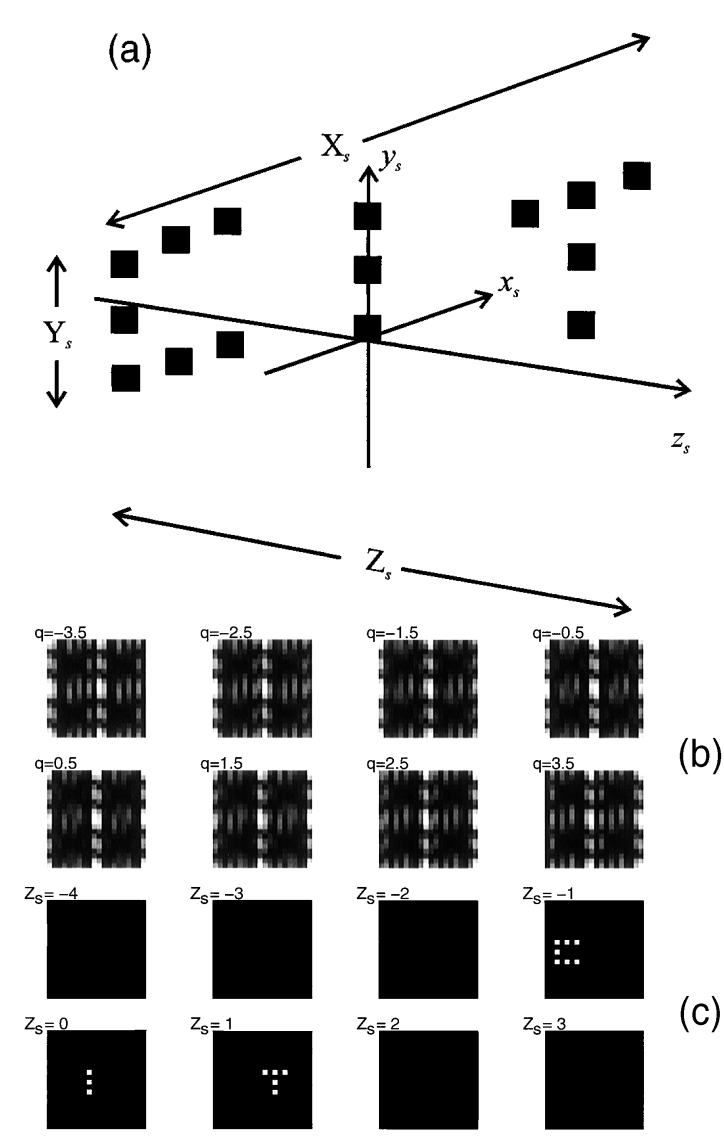

Fig. 2. (a) 3-D source intensity distribution. (b) Part of the 3-D visibility function calculated from the interference gratings of the simulated MSI ( $q=1$ is $q=8.5 \mathrm{~m}$ ). (c) Reconstruction from the complete $3-\mathrm{D}$ complex visibility function on a few planes along the $z_{s}$ axis $\left(z_{s}=1\right.$ is $z_{s}=400 \mathrm{~m}$ ).

As an example we simulate the quasi-monochromatic incoherent light source shown in Fig. 2(a). Each of the three letters is positioned at a different location along the $z_{s}$ axis. Each letter is a collection of point sources of spherical waves. We simulated the MSI operation at a distance $R=10^{5} \mathrm{~m}$ from the source by placing two pinholes at $\mathrm{P}_{1}$ and $\mathrm{P}_{2}$, thus measur- ing the degree of coherence $\mu\left(P_{1}, P_{2}\right)$. We did this by measuring in the far field the complex visibility and phase of the fringes resulting from the interference of the light from $\mathrm{P}_{1}$ and $\mathrm{P}_{2}$ for every point in the space $(\Delta x, \Delta y, q)$. The simulation's parameters are $\lambda=10 \mu \mathrm{m},\left(X_{S}, Y_{S}, Z_{S}\right)=(0.35,0.15,800) \mathrm{m}$, $\left(d_{x}, d_{y}, d_{q}\right)=(2,2,8.5) \mathrm{m}, \hat{r}_{\max }=80.6 \mathrm{~m}, \Delta r_{\min }=$ $1.4 \mathrm{~m}$, and $N=M=K=10$. The resulting 3-D visibility distribution are shown in Fig. 2(b) for a few successive values of $q$. Each value $(i, j, k)$ in this matrix indicates the visibility of the grating obtained when the pinholes are in the $(i, j, k)$ location in the space $(\Delta x, \Delta y, q)$. Finally, the reconstruction of the source intensity in a few transverse planes along the $z_{s}$ axis is shown in Figs. 2(c). These results are obtained by calculation of relation (7). At three transverse planes a different letter was obtained without the background of the unfocused radiation, thus yielding the reconstruction of the object distribution in the 3-D space.

In conclusion, we have developed a formalism and a method for reconstructing the 3-D intensity distribution of a random light source from measurements of the two-point coherence in the far paraxial zone. This method can be applied to a conventional MSI by selection of the pinholes' coordinates $\left(\mathrm{P}_{1}, \mathrm{P}_{2}\right)$ in the degree of coherence $\mu\left(P_{1}, P_{2}\right)$ in the specific way described above; i.e., the pinholes must be situated simultaneously on a radial line emanating from the origin.

This research was supported by the U.S. Army Research Office and the Advanced Research Projects Agency.

\section{References}

1. M. Born and E. Wolf, Principles of Optics, 4th ed. (Pergamon, Oxford, 1970), Chap. 10, p. 491.

2. J. W. Goodman, Statistical Optics, 1st ed. (Wiley, New York, 1985), Chap. 5, p. 157.

3. W. H. Carter and E. Wolf, Opt. Acta 28, 227 (1981).

4. A. J. Devaney, J. Math. Phys. 20, 1687 (1979).

5. I. J. LaHaie, J. Opt. Soc. Am. A 2, 35 (1985).

6. A. T. Friberg, J. Opt. Soc. Am. A 3, 1219 (1986). 